ophthalmoplegia in our patient might be the result of an immune reaction located in the wall of the cavernous sinus. Tests for lupus should be systematically done in every patient affected by painful ophthalmoplegia.

ANTONIO DÁVALOS

JORGE MATIAAS-GUIU

AGUSTII N CODINA

Servicio de Neurologia,

Hospital Valle Hebrón,

Barcelona, Spain

\begin{abstract}
References
' Dornan TL, Espir ML, Gale EA, Tattersall RB, Worthington BS. Remittent painful ophthalmoplegia: the Tolosa-Hunt syndrome? $J$ Neurol Neurosurg Psychiatry 1979; 42:270-5.

${ }^{2}$ Mathew NT, Chandy J. Painful ophthalmoplegia. J Neurol Sci 1970;11:243-56.

${ }^{3}$ Evans OB, Lexow SS. Painful ophthalmoplegia in systemic lupus erythematosus. Ann Neurol 1978;4:584-5.
\end{abstract}

Accepted 14 October 1983

\section{Triphasic EEG discharges in metrizamide encephalopathy}

Sir: The water-soluble contrast agent metrizamide has proved safe and effective in various neuroradiologic procedures.' The adverse effects of this agent differ in some respects from those of other contrast media, and have been well described. ${ }^{2}$ Seizures and transient cerebral symptoms have been seen occasionally, ${ }^{3}$ but increasing attention has been focused on a prolonged toxic encephalopathy with stuttering, obtundation, asterixis, and myoclonic jerks, which on at least one occasion has relapsed after recovery from the initial episode. ${ }^{4-7}$ Various EEG changes have been described after metrizamide injection; ${ }^{89}$ triphasic waves associated with metabolic encephalopathy have recently been considered. ${ }^{6}$ We encountered a patient with toxic encephalopathy after metrizamide myelography whose EEG showed triphasic waves on several occasions. Such patients may provide insight into the mechanisms of triphasic EEG discharges in metabolic encephalopathies.

A 65-year-old female had been healthy except for hypertension treated with salt restriction, but sufferered left leg and foot pain since an automobile accident in $\mathbf{1 9 6 8 .}$ She was referred for implantation of a transcutaneous nerve stimulator after failure of analgesics and antidepressants. Strength was normal except for $4 / 5$ proximal and 3/5 distal weakness in the left leg. Pain and touch sensation were decreased over the lateral and posterior aspects of the left leg, thigh, and buttocks, and the medial aspect of the left foot was hyperaesthetic. Deep tendon reflexes were brisk and symmetric in the arms and hypoactive in the legs, with flexor plantar responses. Laboratory studies were normal. Metrizamide myelography of the thoracolumbar region was carried out, and on the following morning the patient abruptly became confused and agitated. All medication given before or during the myelogram had been discontinued. Neurologic examination was unchanged except for disorientation, monosyllabic responses to stimulation, and asterixis. Complete blood count, blood glucose, serum electrolytes and calcium, liver function tests, arterial blood gases, and cerebrospinal fluid were normal. An electroencephalogram showed continuous triphasic sharp waves of 100-200 $\mu \mathrm{V}$ amplitude and 2-4 $\mathrm{Hz}$ frequency, which were maximal frontocentrally and decreased in amplitude as they extended posteriorly. Occasional theta activity was present in the parietal and occupital areas, but alpha rhythm was absent and background activity was unchanged by stimulation or eye opening. The patient's mental state improved over the next several days, no further studies were undertaken, and she underwent implantation of a dorsal column stimulator. Subsequent EEGs have shown diffuse theta slowing, without triphasic waves of asymmetry.

This patient developed an acute encephalopathy with asterixis and triphasic EEG discharges after metrizamide myelography. No underlying or precipitating medical or neurologic disorder was found, but the subsequent persistence of EEG slowing raises the possibility of a pre-existing mild encephalopathy placing her at risk for this complication. The persistent EEG slowing despite return of the mental state to normal could also reflect a mild but persistent encephalopathy afterward, analogous to the persistent head bobbing reported in two patients by Davis and coworkers. ${ }^{4}$ In these patients, the movement disorder was present for several months after myelography despite normal mentation, and the EEG was diffusely slow in one patient. The mechanism and generator sites of triphasic waves are unknown, but subcortical or thalamic generation has been suggested,' and could be supported by evidence of subcortical origin for other rhythmic EEG patterns, ${ }^{1 "}$ and of the role of thalamic and brain stem centers in the generation of normal EEG rhythms. ${ }^{12}$

$\mathrm{Kaada}^{8}$ studied 79 patients undergoing lumbar myelography, none of whom developed encephalopathy, and observed transient and diffuse slowing in 13 and epileptiform discharges in none. Ropper, Chiappa, and Young ${ }^{9}$ studied prospectively the effects of metrizamide myelography or cisternography in 61 patients, without encephalopathy. Sixteen percent of patients had either paroxysmal discharges or slow activity with some sharp features. This suggested the possibility of diffuse cerebral uptake of metrizamide after lumbar injection, with both irritative and depressive effects.

Vincent and Zimmerman ${ }^{5}$ briefly reported a patient who developed confusion, agitation, tremor, and asterixis after lumbar myelography. The EEG demonstrated generalised slowing and bifrontal triphasic delta transients, and clinical and EEG findings resolved within 48 hours. The authors ascertained the previous occurrence of at least two similar cases, on patient showing triphasic EEG discharges Two patients with transient confusion an $\mathbb{R}$ asterixis after lumbar myelography were reported by Rubin, Horowitz, and Katz: one patient had a normal EEG, while the other tracing showed bilateral and indes pendent paraxysmal sharp transients an slow waves in the posterior temporaf, + parietal, and occipital areas. Bertoni et al. ${ }^{7}$ reported two patients with similar features, one of whom had no EEG and the other a normal tracing when the encephalopathy had virtually resolved. A CT scan of the brain in the former patient demonstrated widespread cerebral uptake of metrizamide.

Two mechanisms for encephalopathic complications of metrizamide myelography were proposed by Bertoni et al.:'? interference with the sodium-potassium ATPase cation pump and competitive inhibition of glucose metabolism as a 2-deoxyglucose analogue. No in vitro inhibition of a partial reaction of the cation pump was found, but metrizamide in concentrations analogous to those after lumbar injection competitively inhibited microbial hexokinase. This finding suggests that the encephalopathy might be due to impaired glucose metabolism.

The clinical and EEG similarities between several recent cases of metrizamide encephalopathy and metabolic derangements typified by hepatic encephalopathy 
might suggest a common mechanism for the generation of triphasic EEG discharges, possibly the impairment of glucose metabolism in subcortical nuclei. Investigation of cerebral glucose metabolism in those patients with metrizamide encephalopathy who have triphasic EEG activity, and study of the effects on the EEG of experimental disturbance of cortical and subcortical glucose metabolism, may clarify the pathogenesis of triphasic waves.

MILES E DRAKE,
CW ERWIN
Division of Neurology,
Department of Medicine,
Department of Psychiatry,
Duke University Medical Center,
Durham,
North Carolina, USA

North Carolina, USA

\section{References}

' Sackett JF, Strother QM, Quaglieri CE, et al. Metrizamide-CSF contrast medium: analysis of clinical applications in 215 patients. Radiology 1977;123: 779-82.

${ }^{2}$ Kieffer SA, Binet EF, Esquerra JV et al. Contrast agents for myelography: Clinical and radiologic evaluation of Amipaque and Pantopaque. Radiology 1978;129:695-705.

${ }^{3}$ Baker AR, Hillman BJ, McLennan JE, et al. Sequalae of metrizamide myelography in 200 examinations. $A J R$ 1978; 130:339-502.

${ }^{4}$ Davis CE, Smith C, Harris R. Persistent movement disorder following metrizamide myelography. Arch Neurol 1982;39:128.

${ }^{5}$ Vincent FM, Zimmerman JE. Metrizamide encephalopathy. Ann Neurol 1980; 7:494.

- Rubin B, Horowitz G, Katz RI. Asterixis following metrizamide myelography. Arch Neurol 1980;37:522.

' Bertoni JM, Schwartzman RJ, Van Horn G, et al. Asterixis and encephalopathy following metrizamide myelography: investigations into possible mechanism and review of the literature. Ann Neurol 1980;9:366-70.

- Kadda B. Transient EEG abnormalities following lumbar myelography with metrizamide. Acta Radiol Suppl (Stockh) 1973;6:222-6.

' Ropper AH, Chiappa KH, Young RR. The effects of metrizamide on the EEG: a prospective study in 61 patients. Ann Neurol 1979;6:222-6.

${ }^{10}$ Bickford RG, Butt HR. Hepatic coma: the electroencephalographic pattern. J Clin Invest 1955;34:790-9.

"Nelson JH, Leffman H. The human diffusely projecting system. Arch Neurol 1963;8:544-56.

12 Anderson P, Andersson SA. Thalamic origin of rhythmic cortical activity. In: Creutzfeldt $O$ (ed): Electrical Activity from the Neuron to the EEG and EMG (Handbook of EEG and Clinical Neurophysiology, vol 2, part C). Amsterdam: Elsevier-North Holland, 1974.

Accepted 14 October 1983

Present address and reprint requests to: Miles E Drake, Jr, MD, Department of Neurology, 463 Means Hall, 1655 Upham Drive, Columbus, Ohio 43210, USA

\section{Notices}

Behavioral Neurology Society. The Third Annual meeting will be held in Boston on 10 April 1984. Further information may be obtained from: Dr Francois Boller, MD, Medical Center, University Drive C, Pittsburgh, Pennsylvania 15240, USA.

Neuro-ophthalmology. Joint world meeting. A joint meeting of the International Society of Neuro-ophthalmology and the Congress of the Study Group of Neuroophthalmology and Neuro-genetics of the World Federation of Neurology will be held in Antwerp, 14-18 May 1984. Information may be obtained from: Professor A Neetens, Academic Hospital, University of Antwerp, Wilrijkstraat 10, 2520 Edegen, Belgium.

International Congress of Physical Medicine and Rehabilitation. The 9th Congress will be held 13-18 May 1984 in Jerusalem, Israel. Information may be obtained from: J. Chaco, MD, PO Box 983, Jerusalem, 91009, Israel.

\section{Eighth International Symposium on Par- kinson's Disease}

Under the auspices of the World Federation of Neurology, Research Committee on Extrapyramidal Disease, this Symposium will be held in New York 9-12 June, 1985. Information may be obtained from Kenneth J Bergmann, MD, Department of Neurology, Mount Sinai School of Medicine, City University of New York, New York, NY 10029, USA.
Evoked Potentials for the Assessment of Central Nervous Disorders: an international symposium and a workshop will be held 27-29 June 1984 at the University Hospital of Lausanne, Switzerland. Information may be obtained from: PA Despland MD, EEG-EMG and Neurophysiological Unit Dept of Neurology, University Hospital - CHUV, 1011 Lausanne, Switzerland. 